\title{
Les réseaux de centres d'excellence : une expérience canadienne
}

\author{
Gilles Charpenet
}

Le Canada vient de s'engager dans une politique scientifique d'identification et de soutien de "Centres d'excellences", privilégiant la collaboration étroite entre les différents partenaires d'un réseau thématique plutôt que le regroupement physique de nombreuses équipes en un seul site. Les thèmes retenus sont clairement finalisés et les coopérations entre l'université et l'industrie sont vivement encouragées. La description de cette politique et de sa mise en cuvre devrait intéresser tous ceux qui ont la charge ou la possibilité d'influer sur les politiques scientifiques de nos pays.

TIRÉS A PART

G. Charpenet.

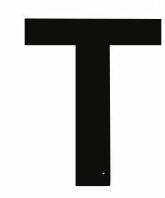

raditionnellement, le Canada a compté, pour assurer son développement et sa prospérité économiques, sur l'exploitation de ses abondantes richesses naturelles : forêts, potentiel hydroélectrique, minerais. Si cette approche a permis d'assurer aux Canadiens un niveau de vie enviable, elle a cependant peu contribué au développement d'une infrastructure propice à la recherche et au développement, non plus qu'à l'épanouissement d'une culture populaire scientifique. L'explosion des connaissances, l'évolution rapide de l'informatique, l'importance croissante de l'innovation technique, la globalisation de l'économie et des marchés, ont contraint le gouvernement canadien à redéfinir sa stratégie. Une plus grande place devait être accordée aux sciences et à la technologie pour permettre au Canada de participer sérieusement à la course internationale à la survie technologique. Cette nouvelle stratégie devait en outre tenir compte de la géographie du pays en assurant un développement régional harmonieux. C'est dans ce contexte qu'en mars 1987 les ministres chargés des Sciences et de la Technologie du gouvernement fédéral canadien, des provinces et des territoires ont signé la Politique nationale en matière de sciences et de technologie - qui devenaient ainsi, pour la première fois dans l'histoire du Canada, une priorité nationale.

\section{InnovAction}

Dans la foulée, le gouvernement fédéral annonçait les détails de sa nouvelle stratégie : InnovAction. Les objectifs en sont l'innovation industrielle et le transfert de la technologie, la mise au point de technologies d'importance pour le Canada, la gestion efficace de toutes les ressources fédérales dans le domaine, le développement des ressources humaines techniques et scientifiques, et l'éveil d'une culture populaire scientifique.

Le programme des Réseaux de centres d'excellence (RCE), annoncé en janvier 1988, fait partie intégrante d'InnovAction. Il en reprend les objectifs et reflète la volonté de promouvoir les liens entre les milieux industriel, académique et gouvernemental (Tableau I). La formation de réseaux devrait aussi contribuer au développement régional en évitant la concentration des compétences et des ressources humaines qu'aurait immanquablement entraînée la création d'instituts physiquement logés sous un même toit. 240 millions de dollars ont été alloués aux RCE par le gouvernement fédéral pour une période de cinq ans. Les provinces et les industries ont été invitées à participer financièrement au programme. Au Québec, c'est par le biais de son Fonds de la recherche en santé (FRSQ) que le gouvernement apporte son appui aux réseaux. Le montant des subventions complémen- 
taires pour les frais indirects pourrait aller jusqu'à 20 millions de dollars, soit près du tiers des fonds des RCE versés aux institutions québécoises.

\section{Mise en cuvre}

Le programme canadien des RCE relève du ministère d'État pour les Sciences et la Technologie qui en a confié la gestion aux trois conseils fédéraux accordant des subventions : le Conseil de recherches médicales, le Conseil de recherches en sciences humaines et le Conseil de recherches en sciences naturelles et en génie. Un comité de direction inter-conseil fut établi pour veiller à la bonne marche du programme. Il est formé des présidents des trois conseils et d'un observateur du ministère. Ce comité a développé les lignes directrices et les politiques des RCE.

Une seconde structure, la direction inter-conseil, fut mise sur pied avec la responsabilité d'orchestrer l'évaluation par les pairs des nombreux projets soumis à l'automne 1988. Ceuxci ont été examinés par un comité international de 23 membres (dont 10 non canadiens) selon quatre critères : l'excellence scientifique (50\%), les liens université-industrie-gouvernement $(20 \%)$, la pertinence industrielle $(20 \%)$ et la structure de gestion $(10 \%)$. Des 158 soumissions,

Tableau 1

\section{OBJECTIFS DU PROGRAMME CANADIEN DES RÉSEAUX DE CENTRES D'EXCELLENCE}

- Stimuler la recherche fondamentale de pointe et la recherche appliquée

à long terme importantes pour le Canada.

- Former et garder au pays des scientifiques et des ingénieurs canadiens d'envergure internationale dans les technologies qui revêtent une importance vitale pour la compétitivité future de l'industrie.

- Intégrer les activités du Canada en matière de recherche et technologie dans des réseaux nationaux auxquels participeront les universités, le secteur privé, le gouvernement fédéral et les provinces selon des critères d'excellence conformes à des normes internationales.

- Créer des liens solides entre les universités et l'industrie pour accélérer la diffusion à cette dernière des connaissances technologiques de pointe.

51 , ne répondant pas aux exigences du programme, ou de moindre qualité, furent éliminées d'emblée, 58 furent examinées par le comité à l'aide de revues externes et 49 reçurent une visite de laboratoire. 107 demandes furent donc classées en trois catégories: (a) doivent être financées : 9 ; (b) recommandées pour financement: 7 ; (c) non recommandées : 91. Finalement, 15 demandes furent approuvées, dont cinq dans le domaine biomédical. Dans l'ensemble, les participants des réseaux sélectionnés sont des chercheurs bien établis, de réputation internationale et subventionnés par les Conseils depuis plusieurs années.
Pour ces 15 demandes, l'aventure n'était pas terminée pour autant. En effet, un comité de mise en œuvre, composé de sept membres provenant d'horizons divers (administration, université, industrie), se vit confier la responsabilité de veiller à la production par chaque réseau d'un accord dit interne. C'est dans ces accords internes, sortes de statuts des réseaux, que sont décrits en détail les structures d'autorité, les structures de gestion, les responsabilités et les pouvoirs des différents acteurs du réseau. On y discute également de propriété intellectuelle, de traitement de l'information confidentielle ou sensible, de conflit d'intérêt, d'emploi, de méca-

\section{Tableau II}

QUELQUES STATISTIQUES CONCERNANT LES CINQ RÉSEAUX ADMINISTRÉS PAR LE CONSEIL DE RECHERCHES MÉDICALES DU CANADA

\begin{tabular}{|lccccc|}
\hline & Montant & Participants & & Centres & Industries \\
\cline { 3 - 6 } & & & Ussociées & $3^{*}$ \\
\hline Ensemble des réseaux & 93500000 & 180 & $16^{*}$ & $15^{*}$ & $3^{*}$ \\
\hline $\begin{array}{l}\text { Base génétique } \\
\text { des maladies humaines }\end{array}$ & 17500000 & 22 & 8 & 1 & 5 \\
\hline $\begin{array}{l}\text { Régénération neurale et } \\
\text { réadaptation fonctionnelle }\end{array}$ & 25500000 & 24 & 13 & - & 2 \\
\hline Génie protéique & 20000000 & 27 & 4 & 6 & 2 \\
\hline Maladies bactériennes & 18200000 & 51 & 7 & 5 & 2 \\
\hline Maladies respiratoires & 12300000 & 56 & 8 & 5 & - \\
\hline
\end{tabular}

* Ces chiffres ne représentent pas les totaux des données de chaque réseau, puisque certains centres participent à plus d'un réseau. 


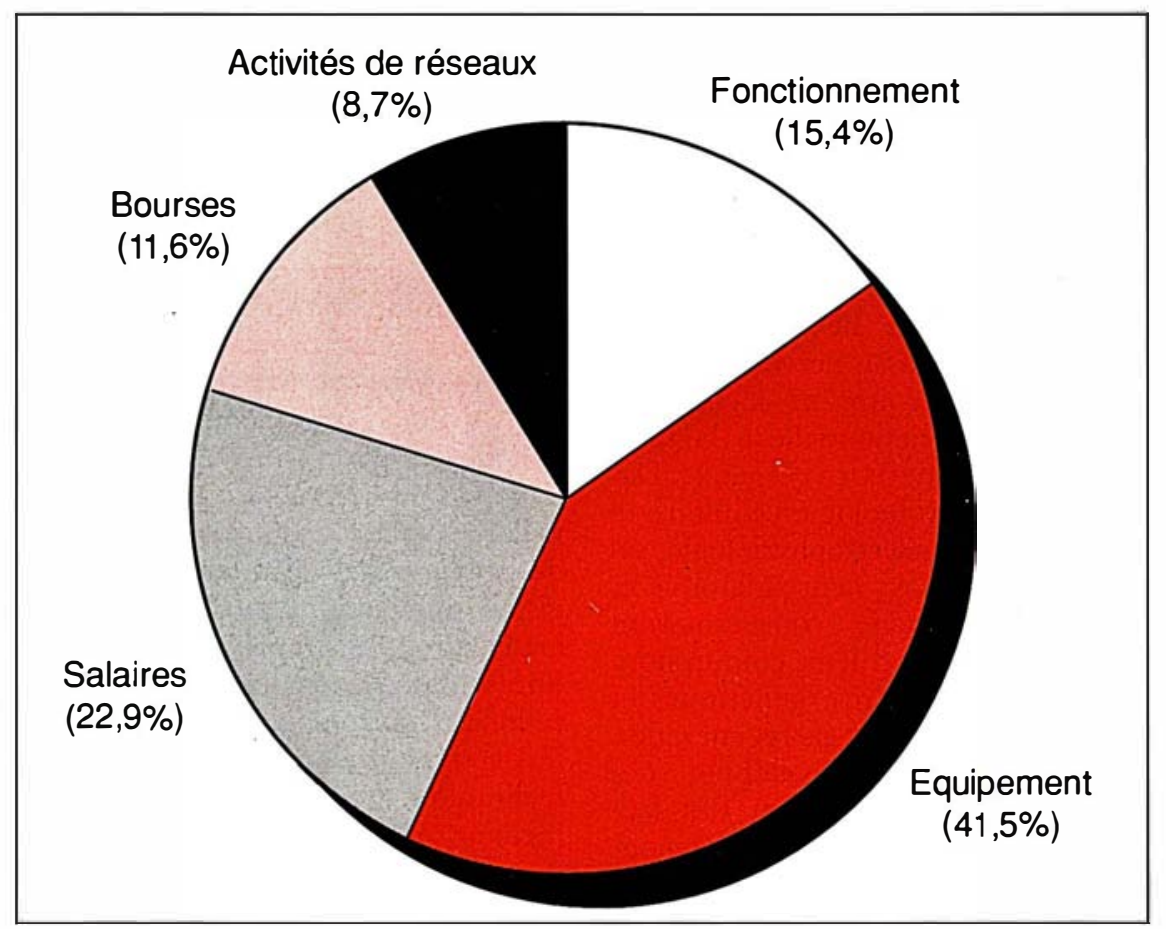

Figure 1. Répartition, en fonction des postes budgétaires majeurs, des fonds fédéraux alloués la première année aux cinq RCE administrés par le Conseil de recherches médicales. La part réservée à l'équipement sera considérablement diminuée dans les années ultérieures. Les montants pour chaque poste (en milliers de dollars) sont les suivants. Fonctionnement : 4574 ; équipement: 12363 ; salaires : 6816 ; bourses : 3451 ; réseau : 2577 .

nismes de résolution de disputes, d'assurance, de rapports scientifiques et financiers. Une fois ces documents jugés acceptables, le financement du réseau peut débuter. La rédaction de ces accords s'est sans doute révélée pénible pour les chercheurs, plus exercés à rédiger des demandes de subventions que des documents à caractère légal. L'exercice était d'autant plus difficile qu'il nécessitait l'obtention d'un consensus entre un grand nombre de parties. Cependant, il est à parier que le temps montrera le bien-fondé de cette exigence.

Chaque réseau est organisé autour d'une unité centrale responsable de l'administration et où l'on retrouve le directeur scientifique, le directeur administratif et les services comptables (généralement assurés par l'université où se situe le bureau administratif). Autour gravitent les centres du réseau qui - sont des laboratoires universitaires, des laboratoires fédéraux ou des industries ayant des employés participant aux travaux du avec chaque réseau (Tableau II). En moyenne, chaque participant recevra 70000 dollars, soit l'équivalent d'une subvention moyenne du Conseil de recherches médicales. Des 5 réseaux médicaux, 3 sont basés à l'université de Colombie britannique (UCB), un à l'université McGill (UMcG) et un conjointement à l'UMcG et à l'université de Montréal (UM).

\section{Des gènes...}

Le réseau de génétique, dirigé par le docteur Michael Hayden, du département de génétique médicale de l'UCB, a pour objet l'élucidation du rôle des gènes dans certaines maladies, en particulier la fibrose kystique, la dystrophie musculaire de Duchenne, le rétinoblastome et divers désordres métaboliques d'origine génétique. Seront également analysés les facteurs génétiques prédisposant à des maladies communes comme des maladies infectieuses, le cancer et l'athérosclérose. D'autres travaux porteront sur la structure de la variation génétique humaine, ou encore sur les gènes responsables de la spécificité tissulaire. Ce réseau regroupe les talents canadiens dans les domaines de la génétique, de la biologie moléculaire, de la biochimie et de la génétique des populations. Les techniques de pointe (hybridomes, animaux transgéniques, clonage...) seront utilisées. Seront également mises à contribution des structures déjà existantes et réputées comme le réseau de médecine génétique du Québec. On tirera également profit de la présence au Canada de populations homogènes comme les familles mennonites du Manitoba, la population chinoise cantonaise de Colombie britannique, et la population québécoise du Saguenay - LacSaint-Jean pour laquelle on dispose d'une banque de données informatisée où sont enregistrés plus de 600000 sacrements de baptêmes administrés depuis 1842 . leurs plans d'intégration des activités de réseau, de communication et de diffusion de technologie af in de convaincre les évaluateurs de l'efficacité des liens entre les différents membres du réseau. Évidemment, le nombre des participants et le nombre des centres ainsi que leur nature varient

\section{... aux maladies} respiratoires...

Le directeur scientifique de ce réseau est le docteur Peter Macklem du département de médecine de l'UMcG. Les recherches proposées 
porteront sur les maladies conduisant à l'obstruction des voies respiratoires. Seront étudiés plus particulièrement : l'asthme, la fibrose kystique, la bronchite chronique et l'emphysème, de même que les maladies pulmonaires dont les causes sont reliées au milieu de travail ou à l'environnement. Cinq thèmes principaux seront abordés : la pharmacologie de composés nouveaux dans le traitement de la fibrose kystique et de l'asthme, le diagnostic, la réadaptation pulmonaire assistée mécaniquement, la santé respiratoire comme fonction de l'environnement et l'analyse par imagerie automatisée de la structure et de la fonction pulmonaires. Ces travaux devraient aboutir à la mise au point de nouveaux médicaments, au développement de meilleures techniques de ventilation pulmonaire et de meilleures techniques de diagnostic rapide des infections pulmonaires, ainsi qu'à l'amélioration des systèmes de ventilation et de purification de l'air dans les immeubles.

\section{... en passant par les neurones...}

Le docteur Albert Aguayo de l'UMcG, et le docteur Yves Lamarre de l'UM se partagent la direction scientifique de ce réseau dont le but est de comprendre les processus biologiques critiques nécessaires à la régénération du tissu nerveux endommagé, et d'accroître et faciliter la réadaptation fonctionnelle à la suite de blessures du tissu nerveux. Des experts en neurobiologie cellulaire et moléculaire, en électrophysiologie, en neurobiochimie, en neuroanatomie et en neuropathologie coordonneront leurs efforts pour mieux comprendre les facteurs cellulaires, génétiques et systémiques facilitant la régénération nerveuse et pour développer des agents pharmaceutiques et des facteurs trophiques capables de promouvoir la survie, la recroissance et la fonction neuronale. Des thérapies expérimentales comme la greffe de tissu nerveux fotal ou de cellules

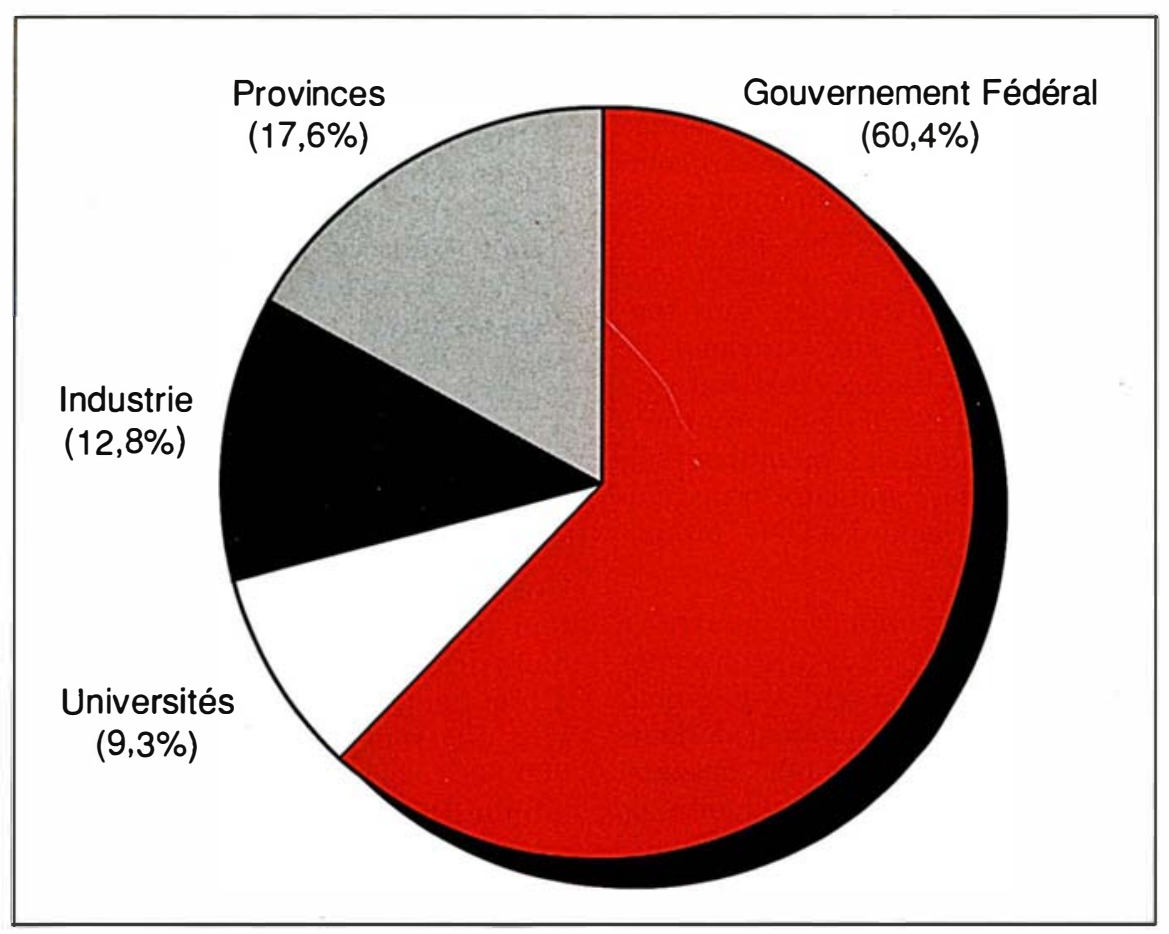

Figure 2. Contributions respectives du gouvernement fédéral, des provinces, de l'industrie et des universités à l'ensemble des cinq réseaux administrés par le Conseil de recherches médicales. Les montants sont les suivants (en milliers de dollars). Gouvernement fédéral : 29781 ; provinces : obtenues par génie génétique seront testées.

\section{... les protéines...}

Le docteur Michael Smith, directeur du laboratoire de biotechnologie de l'UCB, et professeur au département de biochimie, dirige les activités du réseau de génie protéique. Cinq thèmes majeurs seront développés et l'on y fera largement appel à la synthèse protéique ainsi qu'à l'analyse de la structure tridimensionnelle et de la fonction de protéines et de polypeptides d'importance biologique ou économique. Facteurs de croissance, récepteurs, facteurs immunitaires, enzymes (par exemple cellulases) auront ainsi leurs propriétés systématiquement étudiées. Les techniques utilisées sont variées et incluent entre autres la construction de protéines par mutations dirigées, la cristallographie aux rayons $\mathrm{X}$, la résonance magnétique nucléaire et l'analyse structurale assistée par ordinateur. Le transfert de technologie est une caractéristique importante de ce réseau dont les travaux pourraient bien avoir un impact considérable en agriculture, industries de la pêche et de la forêt, ainsi que dans l'industrie pharmaceutique et les soins de santé.

\section{... et les maladies bactériennes}

Le réseau canadien de bactérioses est dirigé par le docteur Robert Hancock du département de microbiologie de l'UCB. Le point focal du réseau est l'interaction hôte-parasite bactérien qui sera étudiée dans plusieurs systèmes biologiques différents l'homme, les animaux d'élevage, les poissons, les plantes - dans le but de développer des modèles expérimentaux d'infection chez l'homme et de parvenir à renforcer les défenses de l'hôte. Les travaux porteront sur les agents pathogènes responsables de maladies comme la coqueluche, les infections pulmonaires dans la fibrose kystique, la gonorrhée, le syndrome du choc septique et les infections nosocomiales. Des agents pathogènes sévissant chez les plantes et les animaux seront également étudiés comme ceux responsables du flétrissement des plantes et de la galle du 
collet, et de la septicémie hémorragique chez le bétail.

\section{Une expérience à suivre}

Le programme des RCE n'est pas un mécanisme visant à accroître le budget des conseils accordant les subventions. Il constitue au contraire une expérience nouvelle que sous-tendent deux hypothèses majeures. Selon la première, au Canada, le modèle d'organisation de la recherche en réseaux est préférable à celui des établissements de recherche centralisés. A ce sujet, le défi des réseaux et des conseils consiste à faire la preuve que les chercheurs peuvent communiquer et collaborer aussi efficacement dans une structure élargie tout en restant compétitifs sur le plan international. La seconde hypothèse est qu'il est souhaitable, possible et bénéfique d'encourager les chercheurs à songer davantage aux applications économiques et sociales de leurs travaux. Dès lors, les réseaux doivent être considérés comme des entreprises scientifiques et commerciales. Dans un contexte d'intense compétition technologique internationale, ce point revêt une importance particulière au Canada où les liens entre le milieu académique et le milieu des affaires n'ont jamais été forts. Les quatre prochaines années seront critiques pour le succès du programme. Les réseaux et les conseils ont désormais la charge de montrer le mérite et la valeur du programme afin que celuici soit renouvelé. Cette expérience devrait également intéresser les administrateurs scientifiques d'autres pays, en particulier ceux de la Communauté économique européenne, qui pourraient voir, dans le modèle des réseaux, une structure bien adaptée au nouveau paysage géopolitique européen

\section{G. Charpenet}

docteur ès sciences cliniques, maitre ès sciences naturelles, Chef. Division du programme des Réseaux de centres d'excellence et du programme des Groupes conseil de recherches médicales du Canada. Ottawa, Ontario, Canada K1A 0W9.

\section{Summary}

Networks of centers of excellence : a Canadian experiment

In January 1988, the Canadian government has launched a new program - under its science and technology InnovAction strategy - to set up networks of centers of excellence. As the funding of these networks is about to start, the author reviews the context, philosophy and objectives of the programme, describes how an international peer review led to the sclection of 15 successful applications, and why these had to fulfil special requirements prior to let the monies flow. A brief overview of the structure of the networks and of the proposed rescarch is given. Finally, the challenges that this experimental programme faces arc discussed.

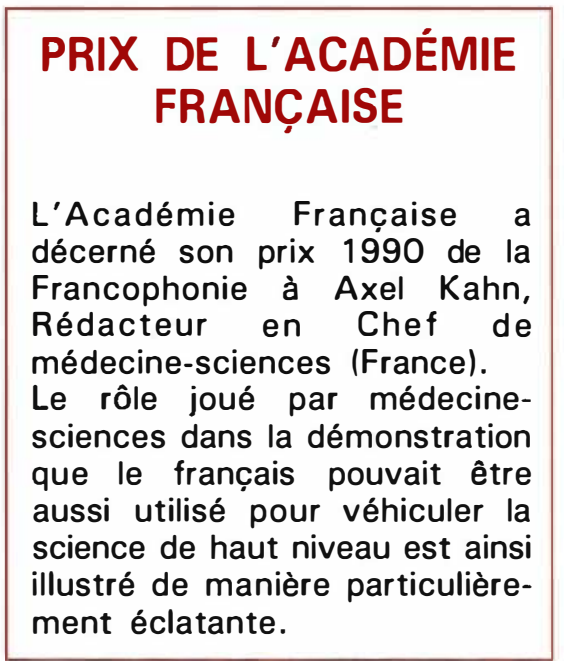

